\title{
Design of a Self-powered Current Measuring Sensor
}

\author{
Changfu $\mathrm{Xu}^{1, \mathrm{a}}$, Shuo Zhang ${ }^{2, \mathrm{~b},{ }^{*}}$, Fengbo Tao ${ }^{1}$, Chengbo $\mathrm{Hu}^{1}$, Jiayuan $\mathrm{Xu}^{1}$, \\ Xin Zhou ${ }^{3}$ and Yu Hong ${ }^{3}$ \\ ${ }^{1}$ Electric Power Research Institute of Jiangsu Electric Power Company, No.1 Paweier Road, \\ Jiangning District, Nanjing, China \\ ${ }^{2}$ Shanghai Jiao Tong University, No.800 Dongchuan Road, Minhang District, Shanghai, China \\ ${ }^{3}$ Suzhou Aquila Solution LTD, Suzhou, China \\ axuchangfu2008@126.com, bzhshdwill@163.com
}

Keywords: Current Measurement, Online Monitoring, Self-powered, Current Transformer.

\begin{abstract}
Current measurement is one of the most important work in online monitoring of the electric system. Self-powered and intelligence are key areas of the current measuring sensor. In this paper, a self-powered current measuring sensor was designed, which realizes synchronous measurement and power supply by one current transformer (CT). The mathematical relationship between the primary side current, secondary side voltage and current of the CT were deduced. The model for line current measurement based on voltage and current signals was constructed, and the overall design of the current measuring equipment was given. Finally, the accuracy of measuring models was verified by experiments.
\end{abstract}

\section{Introduction}

Recently, the development of industries requires more reliable power supplies which have made the transmission and distribution networks more massive. Due to its extensive distribution and complicated running environment, complete state analysis and fault protection is very important. Online condition monitoring is the guarantee to achieve the requirements ${ }^{[1]}$.

Current measurement of lines is an important part of state monitoring because the current value of lines can reflect running state of wires intuitively and many line failures are accompanied by changes of current ${ }^{[2]}$. Therefore, current monitoring is particularly important. As one of the common non-contact current detection methods in the electric power system, the current transformer (CT) based on the principle of transformer is a widely used solution. Since current measurement equipment is mainly applied in a field which has complicated environments, the power supply design of the equipment is very crucial. Studies have shown that the induction power supply ${ }^{[3]}$ is more suitable for this situation. Energy is acquired by the coils of the transformer based on the electromagnetic induction principle.

We find that the CT is not only the core component of the current measurement, but also can be used as a part of the power supply. Many types of research on the measuring theory and the power supply have been reported ${ }^{[4-6]}$, but most of which isolate the two aspects. This paper will explore a solution to integrate the transformer for energy harvesting and the transformer for measuring, thus making measurement and power supply synchronously.

The mathematical relationships between the primary side current, the secondary side voltage and current of the CT were deduced based on the experimental circuit of the power supply. Therefore, the measurable goal could be realized by above algorithm. Then the measurement validity of the proposed sensor design was verified by an experiment.

Such a self-powered design can offer energies through lines directly, thus enabling it to achieve continuous energy supply in complex environment. The equipment has a tight structure and simple capsulation $^{[7]}$, which simplifies the supply circuit design significantly and meets the requirements of small size and low cost. Moreover, the power supply circuit and transmission lines are kept insulated, 
which improves safety and stability. Due to the large number of transmission and distribution lines, the wide application of the smart current sensor will greatly reduce costs in order to achieve distributed measurement.

\section{The Principle of Current Measuring}

The basic working principle of the energy harvesting device ${ }^{[8]}$ is shown in Fig.1. The primary side wire runs through the magnetic core and the current in the wire is $\dot{I}_{1}$. The secondary side current is $\dot{I}_{2}$. The number of turns of the primary coil and the secondary coil are $N_{1}(=1)$ and $N_{2}$. Suppose the transformer working in the linear region.

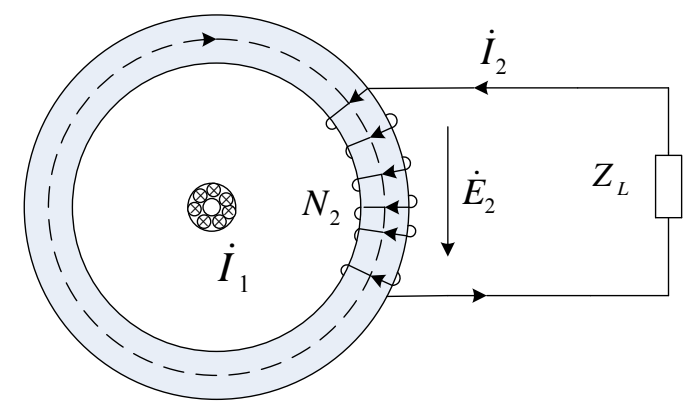

Fig. 1 working principle of the energy harvesting device

According to the basic theory of electromechanics ${ }^{[9]}$, the secondary side voltage of the magnetic core $\left(E_{2}\right)$ could be expressed as:

$$
E_{2}=\sqrt{2} \pi f N_{2} \Phi_{m}
$$

The maximum main flux is $\Phi_{m}=B_{m} S$, where $B_{m}$ is the maximum magnetic induction and $S$ is the net sectional area of the magnetic core. If the magnetic core works in the linear region, the relationship between $B_{m}$ and $H_{m}$ is:

$$
B_{m}=\mu H_{m}
$$

The average length of a magnetic path is expressed by $l$. According to the Ampere circuit law:

$$
H_{m} l=\sqrt{2} N_{1} I_{\mu}
$$

where $I_{\mu}$ is magnetizing current. Combine equations (1), (2) and (3),

$$
E_{2}=\sqrt{2} \pi f N_{2} \Phi_{m}=\sqrt{2} \pi f N_{2} B_{m} S=\sqrt{2} \pi f N_{2} \mu H_{m} S=\frac{2 \pi f N_{2} \mu N_{1} I_{\mu} S}{l}
$$

According to the equilibrium equation of magnetomotive force of the transformer:

$$
\dot{I}_{1} N_{1}+\dot{I}_{2} N_{2}=\dot{I}_{m} N_{1}
$$

If the core loss current is neglected, there's:

$$
\dot{I}_{1} N_{1}+\dot{I}_{2} N_{2}=\dot{I}_{\mu} N_{1}
$$

Substitute the equation (6) into the equation (4), and the relationships between the secondary side voltage, the primary side current and the secondary side current could be known:

$$
E_{2}=\frac{2 \pi f N_{2} \mu\left(N_{1} I_{1}+N_{2} I_{2}\right) S}{l}
$$

Equation (7) could be simplified as:

$$
I_{1}=\frac{E_{2} l}{2 \pi f N_{2} \mu S N_{1}}-\frac{N_{2} I_{2}}{N_{1}}
$$

where $I_{1}$ is the power frequency component, and the secondary side voltage $E_{2}$ and the secondary side current $I_{2}$ contain higher harmonics ( $>3$ ). Therefore, it can conclude that $E_{2}$ and $I_{2}$ have same 
third harmonic phase position. Then, the primary side current could be calculated by measuring the power frequency component of the secondary side voltage and current, thus realizing the goal of current measurement.

\section{Experimental Verification}

To verify the accuracy of measurement principle, an experiment was designed to test the performance of core parts of the sensor. The current source generates standard sinusoidal signals with different amplitudes to simulate different line currents. Firstly, the harmonic relationship between the secondary side voltage and the current was verified. Next, the primary side current was calculated according to signals after the current transformer.

Experimental apparatus includes energy harvesting magnetic core, three-phase power source CL303, rectifier bridge, DC-DC, 470uF capacitor, several resistors and oscilloscope.

The experimental circuit is shown in Fig.2.

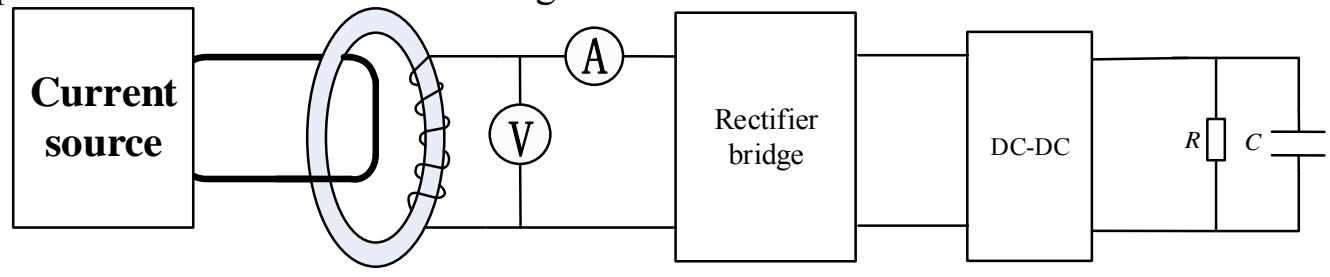

Fig. 2 Experimental circuit

\subsection{Verification of the Harmonic Phase Relationship}

The typical waveforms of the secondary side current and voltage were measured and recorded in the above experimental circuit (Fig.7).

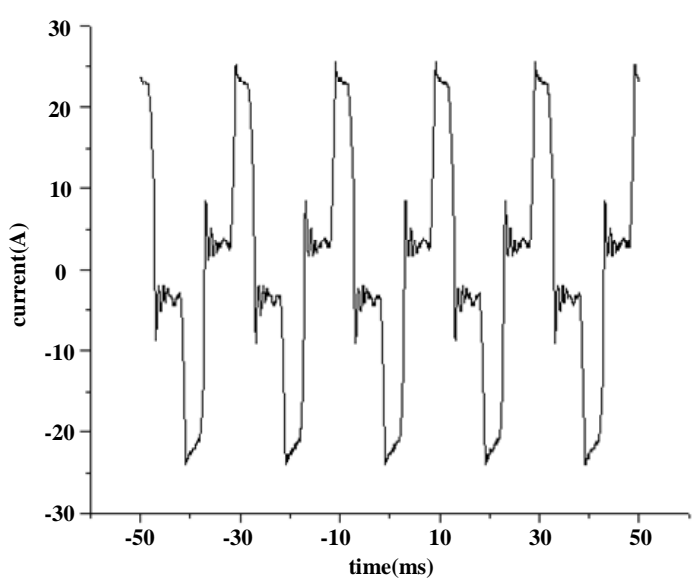

(a) Current waveform

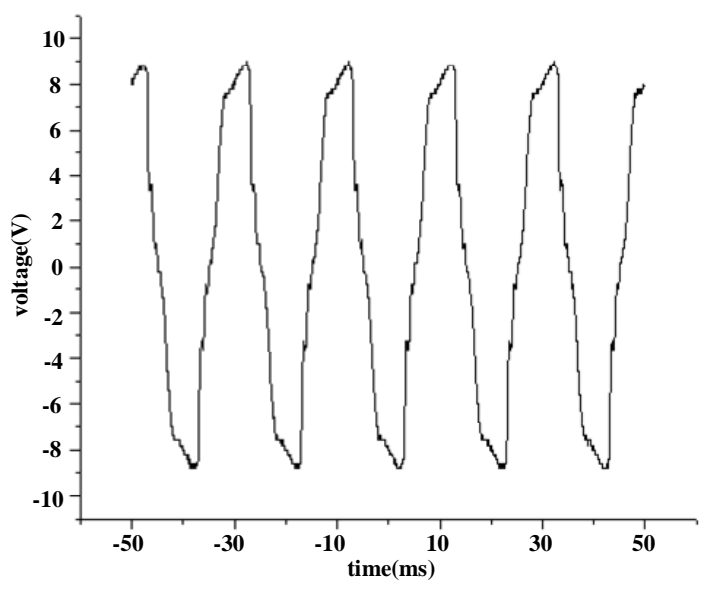

(b) Voltage waveform

Fig.3 Typical waveforms of the secondary side

Amplitudes and phase angles of each harmonic were gained through fast Fourier transform of the above waveforms. Experimental voltage and current before the rectifier bridge were used as the secondary side voltage $E_{2}$ and the secondary side current $I_{2}$. Phase positions of the third harmonic were compared (Table 1).

Table 1. Phase positions of three harmonics

\begin{tabular}{ccc}
\hline I1[A] & $\begin{array}{c}\text { Phase angle of the third } \\
\text { harmonic of E2 }\end{array}$ & $\begin{array}{c}\text { Phase angle of the third } \\
\text { harmonic of I } 2\end{array}$ \\
\hline 200 & 36.65 & 38.57 \\
250 & 40.43 & 41.28 \\
300 & 36.16 & 34.34 \\
350 & 8.17 & 8.62 \\
400 & 5.54 & 3.22 \\
450 & 10.39 & 9.39 \\
500 & 16.72 & 14.56 \\
\hline
\end{tabular}


In Table 1, the FFT analysis concludes basically same phase position of the third harmonic of $E_{2}$ and $I_{2}$, which agrees with theoretical calculation.

\subsection{Calculation Results}

Voltage and current before the rectifier bridge were used as the secondary side voltage and current of the CT. Therefore, the primary side current could be calculated by formula (8) directly.

A $500 \Omega$ load was connected after the rectifier bridge and the DC-DC module. Bring in the measurement data and the calculated results are shown in Table 2.

Table 2 Calculated primary side current based on the secondary side voltage and current [A]

\begin{tabular}{cccc}
\hline Actual value & Calculated value & Fitted value & Error (error/total range) \\
\hline 50 & 60.2258 & 58.5034 & $1.70 \%$ \\
100 & 106.2249 & 107.4502 & $1.49 \%$ \\
150 & 156.3444 & 156.3970 & $1.28 \%$ \\
200 & 203.3174 & 205.3438 & $1.07 \%$ \\
250 & 252.5261 & 254.2906 & $0.86 \%$ \\
300 & 304.0149 & 303.2374 & $0.65 \%$ \\
350 & 355.3479 & 352.1842 & $0.44 \%$ \\
400 & 403.4652 & 401.1310 & $0.23 \%$ \\
450 & 448.4594 & 450.0778 & $0.02 \%$ \\
500 & 497.7144 & 499.0246 & $0.20 \%$ \\
\hline
\end{tabular}

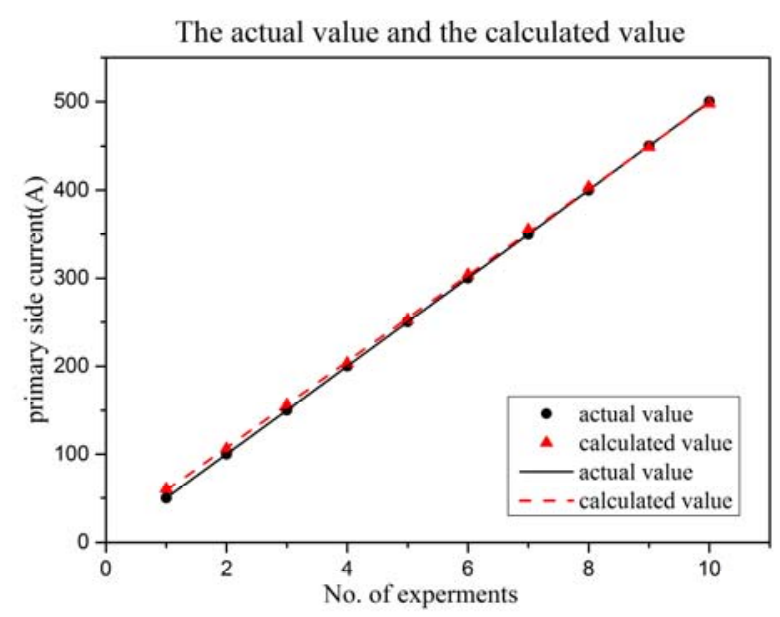

Fig. 4 The actual value and the calculated value

Table 2 and Fig. 4 reveal that the calculated primary side current based on the secondary side voltage and current agree with the actual value basically. The error is within the allowable range, indicating that the theoretical formula is accurate.

\section{Summary}

A self-powered current measuring sensor is designed in this paper. Its measuring principle is introduced, and its accuracy is verified by experiment. Based on the basic electromagnetic induction principle, this sensor uses existing energy harvesting magnetic core for current measurement, realizes synchronous measurement and power supply by one transformer. Such design has positive significance to the research and application of new online monitoring equipment.

The mathematical relationships between primary side current, secondary side voltage and current of the transformer are deduced. The primary side current is calculated according to the secondary side parameters, thus realizing the goal of current measurement. On the secondary side of the current transformer, the voltage and current have same phase positions of the third harmonic, when the current could be gained directly by calculating the power frequency component. Finally, the correctness of the design was proved by experiment. 


\section{Acknowledgements}

This research was funded by a Grant (No. 51307109) from the National Natural Science Foundation of China.

\section{References}

[1]. Yang, Wenxian, R. Court, and J. Jiang. "Research on a novel online condition monitoring technique for induction machinery." Iet International Conference on Power Electronics, Machines and Drives 2012:1-5.

[2]. Yang, Qi Ping, D. F. Xu, and M. Q. Li. "Development of a Power Transmission Line Online Monitoring System." Power and Energy Engineering Conference IEEE, 2011:1-5.

[3]. Du, Lin, et al. "A Novel Power Supply of Online Monitoring Systems for Power Transmission Lines." Industrial Electronics IEEE Transactions on 57.8(2010):2889-2895.

[4]. Moghe, Rohit, F. Lambert, and D. Divan. "A Novel Low-Cost Smart Current Sensor for Utility Conductors." IEEE Transactions on Smart Grid 3.2(2012):653-663.

[5]. Emanuel, Alexander E, and J. /. Orr. "Current Harmonics Measurement by Means of Current Transformers." IEEE Transactions on Power Delivery 22.3(2007):1318-1325.

[6]. Roscoe, N. M., M. D. Judd, and J. Fitch. "Development of magnetic induction energy harvesting for condition monitoring." Universities Power Engineering Conference IEEE, 2009:1-5.

[7]. Liu, Yadong, et al. "A novel high-density power energy harvesting methodology for transmission line online monitoring devices." Review of Scientific Instruments 87.7(2016):653-663.

[8]. Yue, Tianchen, et al. "Power Output Characteristics of Magnetic Core in CT Energy Harvesting Devices." Gaoya Dianqi/high Voltage Apparatus 51.1(2015):18-23 and 29.

[9]. Chapman, Stephen. Electric machinery fundamentals. Tata McGraw-Hill Education, 2005. 